\title{
Neonatal at risk screening and the identification of deafness
}

\author{
P M Watkin, M Baldwin, G McEnery
}

\begin{abstract}
From a cohort of 10686 live births, $322(3 \%)$ were identified as being at risk of a hearing impairment defined as moderate, or worse. These neonates were screened by measurement of auditory brainstem responses. The neonatal at risk screening programme was effective in terms of both yield and cost. The mean age at which hearing aids were fitted was 6 months in the children identified by the neonatal screen. Such a programme is both practicable and useful in a district general hospital. The yield from the neonatal programme was, however, only $43 \%$ of the total number of deaf children eventually identified from the cohort. The need to identify more deaf children by a sensitive infant distraction test screening programme remains.
\end{abstract}

The early identification of deafness is an appropriate objective of child health surveillance programmes. ${ }^{1}$ Children with a severe hearing impairment, or worse, should be identified in infancy, and a case can be made for early detection of children with moderate permanent hearing loss. ${ }^{2}$ The methods by which such identification can be achieved are being critically reviewed. The infant distraction test given at 7 months of age has been recommended as a universal screening test, ${ }^{3}$ and this method used properly can be highly effective in identifying deafness in infancy. ${ }^{4}$ Even when such a test is sensitive, however, the age at which a hearing aid is fitted is often delayed until after the first year of life $\mathrm{s}^{5}$; many now consider that this is too late. The American Joint Committee on Infant Hearing recommended in 1982 that audiological rehabilitation should begin within the first 6 months of life, and this view has been supported by evidence that indicates that fitting a hearing aid at this age improves development of speech. ${ }^{6}$ The screening of neonates for deafness is now therefore a priority for audiology services in the UK.

Measurement of the auditory brainstem response (ABR) is considered the most sensitive method of assessing the auditory acuity of neonates. ${ }^{7}$ Because the procedure is time consuming, however, only selective screening is feasible. The difficulties of identifying children with handicapping conditions by selective screening have been reported in the UK by the Working Group on Risk Registers. ${ }^{8}$ In the USA the Joint Committee on Infant Hearing recommended the use of a risk factor register for selecting the neonatal cohort that would give the highest yield of children with congenital or early onset deafness.
It has been estimated that it should be possible to detect between 50 and $70 \%$ of severely or profoundly deaf neonates from this at risk group. ${ }^{9}$ Most such at risk neonates have been in special care baby units, or have a family history of deafness, or a congenital malformation. Neonatal hearing screening programmes have therefore selected for testing this section of the total birth cohort; it comprises $7-10 \%$ of newborn infants.

The validity of such neonatal screening programmes has been reported from academic departments of audiology and from regional (level 3) neonatal intensive care units. ${ }^{10-14}$ For such programmes to be useful, however, it must be possible to implement them in districts of different characteristics throughout the country. Most health districts have a special care baby unit (a level 1 or 2 neonatal intensive care unit).

In the present study we evaluated a neonatal hearing screening programme that was introduced in 1987 in the east London health district of Waltham Forest, which has a population of around 250000 . Maternity and paediatric care is provided from Whipps Cross Hospital, the special care of neonates being undertaken by a level 2 neonatal intensive care unit. There is also a diagnostic and rehabilitative paediatric audiology service. The selective neonatal hearing screening programme was implemented as an addition, the infant distraction test being retained as the universal screening test of hearing at the age of 7 months.

The evaluation of a new hearing screening programme requires the measurement of the number of deaf children identified by the programme, its cost, and its effect on the remaining screening tests retained for use at other ages. We report here the results of our evaluation on a cohort born between January 1987 and December 1989.

\section{Subjects and methods}

NEONATAL RISK FACTORS FOR DEAFNESS

The American Joint Committee on Infant Hearing At Risk Register was used, with some revisions, to identify those infants at risk for congenital or early onset deafness (table 1). The register was expanded to include those neonates with a gestational age of less than 32 weeks, those who required ventilation for four hours or longer, and those who developed signs of cerebral illness. The American committee recommended the screening of all neonates who had a family history of hearing impairment during childhood. We limited this to include only those neonates who had a family history of permanent deafness in childhood. The medical 
Table 1 Risk factors for congenital or early onset deafness: criteria used by Redbridge and Waltham Forest audiology services

Family history of deafness that required special education or the fitting of a hearing aid in childhood, or of an inherited condition known to be associated with deafness in childhood, even if there is no known deafness in the family

Congenital malformations either of chromosomal, syndromic, or unknown aetiology including craniofacial, branchial arch, and cervical spine dysmorphology; cleft palate; and any malformation of the pinna excluding isolated ear pits and tags

Congenital infections including clinical rubella, cytomegalovirus, toxoplasmosis, herpes, and syphilis; also any maternal history of poxsible infis, herpes, and syphilis; also any mase of possible

Perinatal illness that required admission to the special care baby

unit for one or more of the following reasons:

unit for one or more of the following
(i) Gestation of less than 32 weeks

(i) Gestation of less than 32 weeks

(iii) Birth weight of $1250 \mathrm{~g}$ or less

(iv) Cerebral illness (for example, intraventricular haemorrhage, convulsions, or meningitis)

v) Apnoea requiring ventilation of four hours or more

vi) Jaundice for which exchange transfusion was considered or undertaken

(vii) Administration of aminoglycoside antibiotic in potentially toxic doses

Postsnatal illness: bacterial meningitis or neurological disease

and nursing staff of the special care baby unit were taught how to use the register for identifying at risk babies within the unit. Additional training in how to identify at risk infants who had not been admitted to the special care baby unit was given to community medical officers, health visitors, and general practitioners.

\section{ENROLMENT IN THE PROGRAMME}

Infants who had one or more risk factor were enrolled to the programme. Those who were not admitted to the special care baby unit were examined for risk factors by the health visitor at the new birth visit, and were subsequently reexamined by the community medical officer or general practitioner at the six week health surveillance check. Referrals were also accepted after the paediatric examination of neonates who were born in the maternity unit but not admitted for special care. The audiology service offers an open access policy for parents, and direct parental referrals were accepted into the programme. One person on the special care baby unit was given the responsibility for referring to the programme, but all the staff took part. Babies who were referred to a regional or level 3 unit were enrolled into the programme on their return to the district, irrespective of the results of electrophysiological tests undertaken in the regional unit.

\section{HEARING SCREENING METHOD}

The auditory brainstem response was the method that was used to test hearing. ${ }^{15}$ Where possible neonates were tested at a postconceptional age of 37 weeks or more and when they were in a stable condition. In the unit babies were tested from the low dependency area before they were discharged. Babies were recalled in one to two weeks by the unit staff if they had been discharged before their hearing had been tested. Referrals from the unit were tested in a quiet room adjacent to the unit. Referrals from other sources were tested in the community based hearing assessment centre.

Babies were tested when they were asleep or lying still by an experienced audiologist. Standard silver or silver chloride electrodes were attached to the forehead and the ipsilateral and contralateral mastoid, with 'Blue Tak' and Netelast. ${ }^{16}$ Using a Medelec MS91 and an ST5 stimulator, an alternatively inverted click stimulus was presented at a rate of $50 \mathrm{pps}$ through a standard TDH39 headphone held against the baby's ear. The analysis time was 10 $\mathrm{ms}$ and the filter band width was $200 \mathrm{~Hz}-2000$ $\mathrm{Hz}$. Two repeatable waveforms of 1000 clicks were acquired at $40 \mathrm{~dB}$ normal hearing level (nHL) and $60 \mathrm{dBnHL}$ for each ear. If there was no response the intensity of the stimulus was increased to $80 \mathrm{dBnHL}$.

A clear and repeatable response at $40 \mathrm{dBnHL}$ was judged a 'pass'. The screening test aimed to identify bilateral deafness that was moderate, or worse, and did not aim to identify bilateral deafness that was moderate, or worse, and did not aim to identify unilateral deafness. A response at $40 \mathrm{dBnHL}$ in one ear only was therefore considered to be a 'pass'.

\section{FOLLOW UP OF INFANTS WHO PASSED THE} SCREENING TEST

All the infants who passed the screening test were recalled at 7 months from their expected date of delivery for a behavioural test of hearing. Infant distraction testing, otoscopy, and impedance audiometry were carried out by an experienced audiologist who had not taken part in the neonatal screening. Further audiological assessments were arranged as a result of this 7 month assessment and until an accurate audiological assessment had been achieved by behavioural testing. Arrangements to recall all the infants at 3 years of age for pure tone audiometry have been made, but the results of this test on the entire cohort are not yet available. If an infant did not attend the follow up, the results of the health visitor's distraction test were obtained.

\section{ASSESSMENT OF INFANTS WHO FAILED THE SCREENING TEST}

Neonates who did not have a clear and repeatable auditory brainstem response at $40 \mathrm{dBnHL}$ in at least one ear were assessed audiologically four to six weeks later at the hearing assessment centre. At this appointment electrophysiological testing to threshold was accompanied by impedance audiometry and an assessment of auditory behaviour. Otoscopy and a medical examination was also done. If this examination confirmed moderate, or worse, bilateral hearing loss the infant was immediately referred to the diagnostic and rehabilitation programmes run at the hearing assessment centre. If such a hearing loss was not confirmed, the infant was reviewed at 7 months in the same way as the infants who had initially passed the neonatal screen.

SCREENING OF INFANTS NOT ENROLLED IN THE NEONATAL PROGRAMME

In the absence of risk factors, deafness was identified by checking a list of features of 
auditory behaviour and alerting parents to hearing loss, and by the infant distraction test that was undertaken by the health visitors at 7 months. The coverage of this programme within the district has been almost $90 \%$, and a retrospective analysis of deaf children identified within the district during a 16 year period has confirmed that the distraction test screen identified, among the infants tested, $90 \%$ of those with previously unrecognised severe deafness, or worse. The sensitivity of the test in infants with moderate deafness who received a distraction test rose to $70 \%$ during the early 1980 s. The hearing assessment centre received the referrals from the infant distraction test screen, as well as the reactive referrals resulting from professional and parental concerns.

\section{ANALYSIS OF RESULTS}

Babies in whom the risk factors did not conform to those specified were accepted for screening, but their data were collected separately. A proportion of the neonates admitted to the district's special care baby unit were from neighbouring districts. If they were enrolled in the neonatal screening programme, they were reviewed in Waltham Forest so that the validity of the neonatal screening test and the yield from the unit could be measured. In assessing the value of the programme within the district, data were restricted to residents of Waltham Forest. Details of all the children with moderate bilateral deafness, or worse, who were identified from the district's three year birth cohort were available from the audiology service's database. Information included the numbers diagnosed, the age at diagnosis of deafness, and the age when the hearing aid was fitted from the different screening programmes and from reactive referrals. The age at diagnosis was defined as the age at which a full audiological assessment confirmed the child's hearing impairment. The clinical and health records were examined to confirm the absence of risk factors in any deaf children who had not been enrolled into the neonatal programme.

Although long term monitoring is continuing, the youngest children in the cohort were born in

Table 2 Causes of over-referral for neonatal screening $(n=76)$

\begin{tabular}{lr}
\hline Cause & $N o(\%)$ \\
\hline History of aminoglycoside treatment & $48(63)$ \\
$\quad$ below toxic dose) & $16(21)$ \\
Parental concern & $7(9)$ \\
Isolated ear pits or ear tags & $5(7)$ \\
Inappropriate family history of deafness & \\
\hline
\end{tabular}

Table 3 Sources of referral correlated with risk factors

\begin{tabular}{lcclll}
\hline & $\begin{array}{l}\text { Special care } \\
\text { baby unit }\end{array}$ & $\begin{array}{l}\text { Community } \\
\text { child health } \\
\text { surveillance }\end{array}$ & $\begin{array}{l}\text { Acute } \\
\text { paediatric } \\
\text { service }\end{array}$ & $\begin{array}{l}\text { Audiology } \\
\text { service }\end{array}$ & Parents \\
\hline Family history & 1 & 10 & 7 & 8 & 1 \\
Congenital malformations & 26 & 8 & 1 & 0 & 0 \\
Congenital infection & 0 & 0 & 0 & 0 & 0 \\
Perinatal illness & 251 & 7 & 1 & 0 & 0 \\
Postnatal illness & 0 & 0 & 1 & 0 & 0 \\
\hline Total & 278 & 25 & 10 & 8 & 1 \\
\hline
\end{tabular}

December 1989. This is an important limitation of the study. Incomplete ascertainment inevitably biased the mean age at diagnosis and at fitting of the hearing aid of the deaf children identified since the neonatal screening programme. The degree of under ascertainment was estimated by comparing the prevalence of deafness identified from the cohort by the end of the study with the period prevalence of moderate bilateral deafness, or worse, that had previously been reported in children from the district. $^{5}{ }^{17}$

\section{Results}

In the three year period from January 1987 to December 1989, 12186 babies were born in the maternity unit at Whipps Cross Hospital. During the same period there were 10686 live births to residents of Waltham Forest, 796 of whom were not born in the district's maternity unit. Babies referred for neonatal screening totalled 398 , but 76 did not conform to the risk factors specified in the register (table 2). Forty eight of these referrals were made during the initial period of the study and consisted of neonates who had been treated with aminoglycosides, but not in potentially toxic doses. This ceased after an early audit, but the remaining 28 were made during the course of the study. Although they were all tested and followed up, none had a hearing impairment and they were excluded from the results of the evaluation.

In all, 322 neonates were admitted to the programme, and 278 were born to residents of Waltham Forest. They represented $2 \cdot 6 \%$ of the live births to residents of the district during the three years. Of the neonates admitted to the programme, 44 (14\%) were enrolled from sources other than the special care baby unit (table 3).

The mean gestational age of the babies who were screened was 35 weeks, with a mean chronological age of 6 weeks at the time of the initial test. The outcome is shown in figure 1. Of the 322 babies tested only those $47(15 \%)$ who failed the screen at $40 \mathrm{dBnHL}$ in both ears were referred for diagnostic audiological assessment.

After the diagnostic assessments 12 infants of the 322 screened $(4 \%)$ were identified as having permanent bilateral deafness which was moderate, or worse, in the better ear. The comparative numbers who had the different risk factors is shown in table 4 . Of the 874 babies admitted to the special care baby unit, eight $(0.9 \%)$ had such a hearing loss, but because less than a third of these infants required a neonatal hearing test, the number was eight of the 278 (3\%) from the unit who were enrolled into the programme. Of the six infants whose deafness resulted from perinatal illness, all had at least three perinatal risk factors, and only one was born with a gestational age of more than 32 weeks. Of the 44 infants referred from other sources in only four $(9 \%)$ was deafness identified by the screening test.

Of the 275 infants who passed the initial screen, $88 \%(n=243)$ were re-examined audio- 


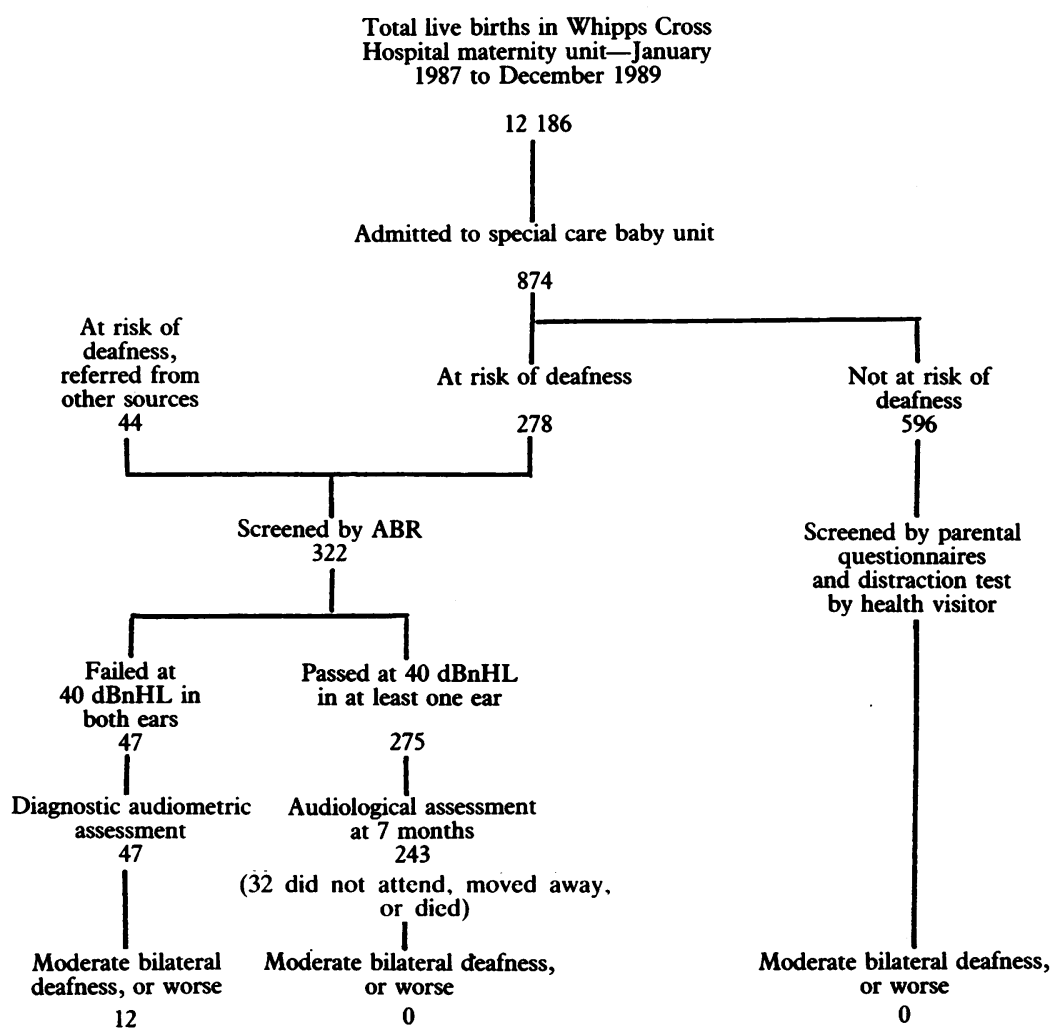

Figure 1 Flow diagram of the neonatal screening programme and outcome.

Table 4 Incidence of deafness correlated with different risk factors

\begin{tabular}{lccc}
\hline & $\begin{array}{l}\text { No } \\
\text { screened }\end{array}$ & $\begin{array}{l}\text { No with } \\
\text { moderate or } \\
\text { bilateral deafness }\end{array}$ & $\begin{array}{l}\text { Incidence of } \\
\text { deafness as } \\
\text { percentage of } \\
\text { No screened }\end{array}$ \\
\hline Family history & 27 & 2 & 7 \\
Congenital malformations & 35 & 4 & 11 \\
Congenital infection & 0 & 0 & 0 \\
Perinatal illness & 259 & 6 & 2 \\
Postnatal illness & 1 & 0 & 0 \\
\hline Total & 322 & 12 & 4 \\
\hline
\end{tabular}

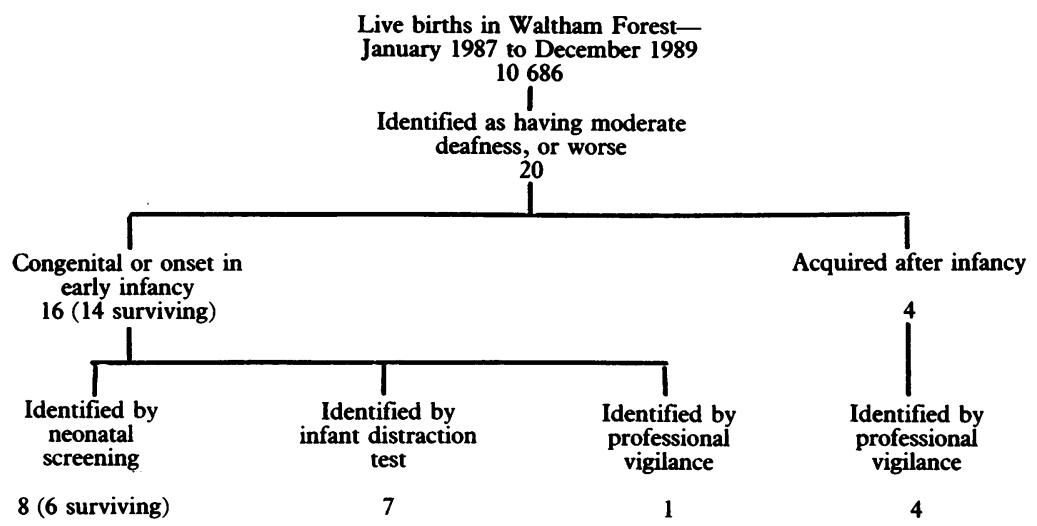

Figure 2 Methods of identification of children with moderate deafness, or worse, who were resident in Waltham Forest and born from January 1987 to December 1989. logically at 7 months. None had moderate deafness, or worse, bilaterally, confirming that the ABR test was $100 \%$ sensitive. The test had a specificity of $89 \%$.

The hearing of the infants was not, however, readily measured at the 7 month examination; 134 of the 322 infants who had received a neonatal hearing test $(42 \%)$ were not readily testable by the distraction test at a corrected age of 7 months. Of these, all but 13 were successfully tested later in infancy when their auditory behaviour had matured. These 13 children had either an additional physical handicap or developmental retardation such that audiological assessment by behavioural methods was difficult, even for an experienced audiologist. The measures of validity of the neonatal screening test were therefore only completed after almost half the infants had been audiologically reexamined on more than one occasion. A few required multiple re-examinations. Of the 12 infants who were identified as by the neonatal screen being permanently deaf, two died later in infancy, and five had an additional handicap. In the remaining five, however, deafness was the only appreciable impairment.

The effectiveness of the neonatal screening programme within Waltham Forest was assessed. During the three year period of the study there were 10686 live births to residents of the district. A total of 20 children with a moderate hearing loss, or worse, in the better ear have been identified from this cohort by hearing screening programmes or by professional and parental vigilance (fig 2). Two of the children died in infancy. The prevalence of this degree of deafness within the cohort was therefore $1 \cdot 7 / 1000$ compared with the previously reported prevalence of $1 \cdot 9 / 1000$. There was slight under ascertainment during the period of the study and examination of the degree of hearing loss identified in children in the cohort suggested that two children with moderate deafness had not been identified by the end of the study.

The routes through which the children with this degree of deafness were identified are shown in fig 2 . Of the 14 surviving children with deafness in infancy, six (43\%) were identified from the 278 who underwent neonatal screening. The eight children who were subsequently identified had not been considered to be at risk of deafness during the neonatal period. A retrospective examination suggested that three of these eight were at risk. One had congenital rubella, but the history was obscure and there were no other physical signs. The two others had a dominantly inherited deafness, but in both cases the family history was not known until after audiological examination of other members of the family; initial direct question-

Table 5 The mean (range) age at diagnosis and fitting of hearing aid (months)

\begin{tabular}{|c|c|c|c|c|}
\hline & $\begin{array}{l}\text { No of children } \\
\text { with deafness } \\
\text { identified }\end{array}$ & $\begin{array}{l}\text { No of children } \\
\text { fitted with } \\
\text { with hearing aid }\end{array}$ & Age at diagnosis & $\begin{array}{l}\text { Age at fitting of } \\
\text { hearing aid }\end{array}$ \\
\hline $\begin{array}{l}\text { Neonatal screening programme } \\
\text { Infant distraction programme or vigilance }\end{array}$ & $\begin{array}{l}6 \\
8\end{array}$ & $\begin{array}{l}5 \\
7\end{array}$ & $\frac{2}{12 \cdot 5}(9 \cdot 5-30)^{*}$ & $\begin{array}{l}5 \cdot 7(1 \cdot 5-8) \\
16(11-34)\end{array}$ \\
\hline
\end{tabular}

*The range is wide because a child was included who was identified in infancy and then went abroad; the diagnosis. was not confirmed until his return. 
ing had not uncovered the presence of deafness within the family. None could have reasonably been identified by the use of the at risk register.

The mean age at diagnosis of the deafness was calculated for those children identified from the different screening programmes (table 5). Two of the children had not been fitted with hearing aids by the conclusion of the study. In both cases the parents initially did not accept the need for the child to wear a hearing aid, although in both cases the presence of the deafness was acknowledged and follow up arrangements had been made.

The mean age at diagnosis for those identified by the neonatal screen was significantly lower than for those identified either by the infant distraction test screen or by vigilance $(t=4.01$, $\mathrm{df}=12, \mathrm{p}<0.01)$. Consequently the mean age at which hearing aids were fitted for the children identified by at risk screening was significantly reduced $(t=2 \cdot 87, \mathrm{df}=10, \mathrm{p}<0.02)$.

\section{Discussion}

The results of the study confirmed the sensitivity of the ABR with a lower stimulus intensity of 40 $\mathrm{dBnHL}$ for identifying from the neonates tested those who had moderate deafness, or worse. From the three year cohort, six of the 14 surviving children with this degree of deafness present in infancy (43\%), were identified by the neonatal screening of those with risk factors.

The selection of the neonates at risk who required screening was highly effective. By selecting and screening 278 neonates from a birth cohort of over $10000,43 \%$ of the deaf children were identified. Of the eight deaf infants who were identified subsequently, seven were identified by the infant distraction screening programme, and one by professional vigilance. To identify the seven children by distraction testing, however, over 9000 children were screened within the district.

The effectiveness of the neonatal screening programme resulted from successfully identifying those neonates with risk factors for congenital or early onset deafness. It has previously been recommended that all admissions for special care should be screened. As in other districts this was $7 \%$ of the hospital births. By selecting only those neonates with specific risk factors from within the level 2 unit, however, only a third of the admissions to the special care unit received a hearing test.

If all babies discharged from the unit had been screened, the number diagnosed would not have increased. Although the largest number was obtained from the special care baby unit, the screening of those neonates referred from other sources was the most efficient. As a percentage of those screened, the number was more than three times greater among those referred from other sources. The highest yield as a percentage of those screened was among those neonates referred with a congenital malformation, or with a family history of deafness. The community child health service played an important part in referring such infants, and if such a programme is to be effective the community child health professionals must continue to refer at risk neonates. If general practitioners are to make a contribution, further education within the district is required.

Not all deaf children who are at risk can be identified from a neonatal risk factor register. Of the eight deaf infants who were not referred for neonatal screening, a retrospective examination of the causes of their deafness confirmed that three of them were at risk. Using a wider set of risk factors would not have identified them, however, and they could not have reasonably been identified as being at risk within the neonatal period. This discrepancy may explain why some authors have suggested that a higher proportion of deaf infants are identifiable from an at risk register ${ }^{9}$; in reality not all these infants will be identified neonatally.

The efficiency of the neonatal screen was reflected in the cost/hearing impairment detected. The ABR screening and the diagnostic testing of those who failed the screen were done by an audiological scientist during a weekly session in the special care baby unit and a fortnightly session in the community hearing assessment centre. With four neonatal screenings, or two diagnostic ABRs being done during each session, the programme was sustainable, even allowing for periods of absence. Taking account of the costs to the health authority of employing an audiological scientist for these sessions, but excluding equipment costs and other overheads, the cost of identifying a hearing impairment in a child by the neonatal screening programme was just under $£ 1250$. Currently (1991) the same cost would be incurred by employing a grade $A$ scientist, or an audiology technician at senior grade (MTO 3), to undertake the ABR testing. This is less than the national cost of $£ 4000$ suggested by the Institute of Hearing Research in Nottingham. The Nottingham programme provides ABR screening for neonates admitted to level 3 units and employs a fulltime nurse ( $G$ grade) to undertake the testing (A Davis, Institute of Hearing Research, Nottingham, personal communication). In most districts, by successfully identifying those neonates with risk factors from within a level 1 or 2 unit, most neonates admitted for special care will not require screening, and a beneficial yield can be obtained at lower cost.

The assessment of hearing by behavioural methods was difficult in many of the infants at risk, and half the deaf children identified by the neonatal screen had additional physical handicaps that would have delayed the introduction of audiological rehabilitation if their deafness had been identified solely on behavioural assessment. An early failure on ABR screening assisted in the diagnosis of deafness in these children, and hearing aids were fitted to the children identified by neonatal screening at a mean age of 6 months. The mean age of fitting hearing aids to the children who were subsequently identified was 16 months, and because of the lack of long term monitoring of the cohort, not all the children with moderate bilateral deafness, or worse, will have yet been identified. Their later identification will further increase the mean age at which deafness is 
confirmed in those not identified by the neonatal at risk screening programme.

The introduction of the neonatal hearing screen has therefore been of value to the district. Within districts with similar characteristics the implementation of such a programme can successfully identify just under half of the children with congenital or early onset deafness, and is relatively cost effective. Most deaf children, however, must still be identified by the traditional hearing screening programmes, or by sensitive parental and professional observation.

We thank Dr John Stroobant for devising the at risk criteria, Steven Mason and Bernice Bradford for instruction in ABR methods, and Adrian Davis for costing the programme.

Haggard $M$. Hearing screening in children-state of the art(s). Arch Dis Child 1990;65:1193-8.

2 Sancho J, Hughes E, Davis A, Haggard M. Epidemiological basis for screening hearing. In: McCormick B, ed. Paediatric audiology $0-5$ years. London: Taylor and Francis, 1988: audiol $1-35$.

3 Advisory Committee on Services for Hearing Impaired People (ACSHIP). Final report of the sub-committee appointed to consider services for hearing impaired children. London: DHSS, 1981

4 McCormick B, Wood S, Cope Y, Spavins FM. Analysis of records from an open access audiology service. $\mathrm{Br} \mathcal{F}$ Audiol 1984;18:127-32.

5 Watkin PM. The age of identification of childhood deaf- ness-improvements since the 1970s. Public Health 1991; 105:303-12.

6 Markides A. Age at fitting of hearing aids and speech intelligibility. Br $\mathcal{F}$ Audiol 1986;20:165-8.

7 Mason S, McCormick B, Wood S. Auditory brainstem response in paediatric audiology. Arch Dis Child 1988;63: response
465-78.

8 Oppe T. At risk registers. Appendix F. In: Court SDM chairman. Fit for the future. London: HMSO, 1976: 413-5.

9 Gerber SE. Review of a high risk register for congenital or early onset deafness. $\mathrm{Br}$ F Audiol 1990;24:347-56.

10 Alberti PW, Hyde ML, Rike K, Corbin H. Abramovitch S An evaluation of BERA for hearing screening in high risk neonates. Laryngoscope 1983;93:1115-21.

11 Galambos R, Hicks GE, Wilson MJ. The auditory brain stem response reliably predicts hearing loss in graduates of a tertiary intensive care nursery. Ear Hear 1984;5:254-60.

12 Stevens JC, Webb HD, Hutchinson J, Connell J, Smith MS Buffin JT. Click evoked otoacoustic emissions compared with brainstem electric response. Arch Dis Child 1989;64: 1105-11.

13 Scanlon PE, Bamford JM. Early identification of hearing oss: screening and surveillance methods. Arch Dis Child 1990;65:479-85.

14 Davis AC, Sancho J. Screening for hearing impairment in children: a review of current practice with special reference to the screening of babies from special care baby units for to the screening of babies from special care baby units for severe/profound hearing impairments. In: Gerber SE, Mencher GT, eds. International perspectives on communic

15 Mason SM, Adams W. An automated micro-computer based Mason SM, Adams W. An automated micro-computer based
electric response audiometry system for machine scoring of auditory evoked potentials. Clinical Physics and Physiological Measurement 1984;5:219-22.

16 Bradford BC, Baudin J, Conway MJ, Hazell JWP, Stewart $A L$, Reynolds EOR. Neural hearing loss in very pre-term infants by brainstem audiology evoked potentials. Arch Dis Child 1985;60:105-9.

17 Watkin PM, Baldwin M, Lacide S. Parental suspicion and identification of hearing impairment. Arch Dis Child 1990;65:846-50. 